\title{
Two-dimensional examples of rank-one convex functions that are not quasiconvex
}

\author{
by M. K. Benaouda (Lille) and J. J. Telega (Warszawa)
}

\begin{abstract}
The aim of this note is to provide two-dimensional examples of rank-one convex functions which are not quasiconvex.

1. Introduction. In the study of equilibrium problems of nonlinear elasticity by the direct method of the calculus of variations the sequential weak lower semicontinuity (s.w.l.s.c.) of the functional of the total potential energy is required. The vector case involves integral functionals of the form

$$
J(u)=\int_{\Omega} f(x, u(x), \nabla u(x)) d x,
$$

where $u: \Omega \subset \mathbb{R}^{n} \rightarrow \mathbb{R}^{m}$ and $\nabla u(x)$ is identified with an $n \times m$ matrix. To ensure the existence of a minimizer, $f$ has to satisfy some conditions $[1$, $3,4]$. The most important is the quasiconvexity of $f$ introduced by Morrey [6] (cf. also $[4,5]$ ). Morrey [6] showed that the quasiconvexity of $f$ is necessary and sufficient for $J$ to be sequentially weak-* lower semicontinuous on $W^{1, \infty}(\Omega)^{m}=\left[W^{1, \infty}(\Omega)\right]^{m}$. Ball and Murat [2] weakened the notion of quasiconvexity, introducing the so-called $W^{1, p}$-quasiconvexity. The quasiconvexity in the sense of Morrey [6] is simply the $W^{1, \infty}$-quasiconvexity and is equivalent to the $W^{1, p}$-quasiconvexity provided that $f$ is bounded from below and satisfies a natural growth condition (cf. [4] and Def. 2.1 below).

When applied to specific cases the quasiconvexity reveals its essential disadvantage: one has to verify an integral condition. Easier to check is a particular case of quasiconvexity, namely polyconvexity (cf. $[3,4])$. The last notion is of algebraic nature and is very useful for a class of stored energy functions in finite elasticity. Weaker than quasiconvexity is rank-one convexity $[4,5,8]$. When $m=1$ or $n=1$ all these notions coincide and are equivalent to convexity. It seems that the gap between quasiconvex and rank-
\end{abstract}

2000 Mathematics Subject Classification: 49J40, 49J45.

Key words and phrases: quasiconvexity, rank-one convex function. 
one convex functions is relatively narrow. Šverák [8] showed that for $m \geq 3$ and $n \geq 2$ rank-one convexity does not necessarily imply quasiconvexity (cf. also [7]).

The aim of this note is to study the two-dimensional case with $m=n=2$. We provide counterexamples extending the results of Šverák to this case.

2. Preliminaries. Let $M^{n \times m}$ denote the set of real $n \times m$ matrices, $m, n \in \mathbb{N}$. Let $\Omega \subset \mathbb{R}^{n}$ be a bounded domain. We denote by $W^{1, p}(\Omega)^{m}=$ $W^{1, p}\left(\Omega, \mathbb{R}^{m}\right)$ the usual Sobolev space, $1 \leq p<\infty$. If $a \in \mathbb{R}^{n}, b \in \mathbb{R}^{m}$, then $a \otimes b$ denotes the tensor product: $(a \otimes b)_{i j}=a_{i} b_{j}$. The canonical basis is $\mathbb{R}^{n}$ is denoted by $\left\{e_{i}\right\}_{1 \leq i \leq n}$. The gradient of a vector $u$ is denoted by $\nabla u$ and $|D|$ stands for the Lebesgue measure of a set $D$.

We now recall the definitions of useful notions.

Definition 2.1 (Dacorogna [4], Morrey [6]). A measurable and locally integrable Borel function $f: M^{n \times m} \rightarrow \mathbb{R}$ is quasiconvex if

$$
\int_{D} f(A+\nabla u(x)) d x \geq f(A)|D|
$$

for every $A \in M^{n \times m}, u \in W_{0}^{1, \infty}(\Omega)^{m}$ and every open bounded subset $D \subset \mathbb{R}^{n}$.

Definition 2.2 (Dacorogna [4]). A function $f: M^{n \times m} \rightarrow \overline{\mathbb{R}}=\mathbb{R} \cup\{+\infty\}$ is said to be rank-one convex if

$$
f(\lambda A+(1-\lambda) B) \leq \lambda f(A)+(1-\lambda) f(B)
$$

for every $\lambda \in[0,1], A, B \in M^{n \times m}$ with $\operatorname{rank}(A-B) \leq 1$.

REMARK 2.3. (i) In Definition 2.1 the space $W_{0}^{1, \infty}(\Omega)^{m}$ of test functions can be replaced by $C_{0}^{\infty}(\Omega)^{m}$. If relation (2.1) holds for one nonempty domain $D \subset \mathbb{R}^{n}$, then it holds for an arbitrary bounded subset of $\mathbb{R}^{n}$ (see [4]).

(ii) If $f$ is a rank-one convex function then the function $t \mapsto f(A+t a \otimes b)$ is convex in $t$ for every $A \in M^{n \times m}$ and every $a \in \mathbb{R}^{n}, b \in \mathbb{R}^{m}$. It is known that every rank-one convex function is continuous in its domain and for $f \in C^{2}$ inequality (2.2) is equivalent to the Legendre-Hadamard condition (or ellipticity condition) (see $[4,8]$ ).

Definition 2.1 can be reformulated in terms of periodic functions (see $[4,8])$.

Lemma 2.4. A continuous function $f: M^{n \times m} \rightarrow \mathbb{R}$ is quasiconvex if and only if

$$
\int_{[0,1]^{n}} f(A+\nabla u(x)) d x \geq f(A)
$$

for every $A \in M^{n \times m}$ and every smooth periodic function $u: \mathbb{R}^{n} \rightarrow \mathbb{R}^{m}$. 
3. Examples of rank-one convex functions that are not quasiconvex. Throughout this section we assume that $n=m=2$ and we set $M^{2}=M^{2 \times 2}$. Definitions 2.1 and 2.2 are certainly global, similarly to the notion of convexity of a function. It thus suffices to provide an example of a rank-one convex function which is not quasiconvex at a certain point.

TheOREM 3.1. Let $h: \mathbb{R}^{2} \rightarrow \mathbb{R}$ be a continuous function, homogeneous of degree one with $h\left(e_{2}\right)>0$. Let $g: M^{2} \rightarrow \mathbb{R}$ be defined by

$$
g(A)=h\left(a_{11}, a_{21}\right)+h\left(a_{12}, a_{22}\right)
$$

where $A=\left[a_{i j}\right], 1 \leq i, j \leq 2$. Then $g$ is rank-one convex at zero but is not quasiconvex at zero.

Proof. Take arbitrary vectors $a, b \in \mathbb{R}^{2}$. We have to show that the function $k(t)=g(t a \otimes b)$ is convex. Since $h$ is homogeneous of degree one, so is $g$. Thus $k(t)=t g(a \otimes b)$ is a convex function.

Set $u\left(x_{1}, x_{2}\right)=(0, \vartheta(x))$, where $\vartheta(x)=\sin \frac{3 \pi}{2} x_{2}$.

A simple computation yields

$$
\nabla u(x)=\left[\begin{array}{cc}
0 & 0 \\
0 & \frac{3 \pi}{2} \cos \frac{3 \pi}{2} x_{2}
\end{array}\right]=\left(\frac{3 \pi}{2} \cos \frac{3 \pi}{2}\right) e_{2} \otimes e_{2} .
$$

Thus

$$
g(\nabla u(x))=g\left[\left(\frac{3 \pi}{2} \cos \frac{3 \pi}{2} x_{2}\right) e_{2} \otimes e_{2}\right]=\left(\frac{3 \pi}{2} \cos \frac{3 \pi}{2} x_{2}\right) g\left(e_{2} \otimes e_{2}\right) .
$$

Integration yields

$$
\int_{[0,1]^{2}} g(\nabla u(x)) d x=g\left(e_{2} \otimes e_{2}\right) \int_{0}^{1} \int_{0}^{1} \frac{3 \pi}{2} \cos \frac{3 \pi}{2} x_{2} d x_{1} d x_{2}=-g\left(e_{2} \otimes e_{2}\right) .
$$

The assumption $h\left(e_{2}\right)>0$ implies that

$$
\int_{0}^{1} \int_{0}^{1} g(\nabla u(x)) d x<0=g(0) .
$$

This means that $g$ is not quasiconvex at zero.

REMARK 3.2. (i) The above theorem remains valid under the assumption that the function $h$ is homogeneous of even degree.

(ii) An explicit example of the function $h$ is provided by

$$
h(x, y)= \begin{cases}\frac{x^{3} y}{x^{2}+y^{2}}+y & \text { if } x \neq 0 \text { and } y \neq 0 \\ y & \text { if } x=0 \text { or } y=0 .\end{cases}
$$

It can be easily verified that $h$ is continuous, homogeneous of degree one and $h\left(e_{2}\right)=h(0,1)=1>0$. 
4. On a result of Šverák [8]. It is well known that for quadratic functions, rank-one convexity is equivalent to quasiconvexity. Šverák [8] devised a construction of some rank-one convex functions. However, the question whether these functions are quasiconvex or not seems to be open.

Lemma 5.1 of Šverák [8] implies the following result.

Lemma 4.1. Let $H: \mathbb{R}^{2} \rightarrow \mathbb{R}$ be the bilinear form defined by $H(x, y)=x y$ and let $g: M^{2} \rightarrow \mathbb{R}$ be given by

$$
g(A)=a_{11} a_{22}+a_{12}^{2}+a_{21}^{2}, \quad A=\left[a_{i j}\right]_{1 \leq i, j \leq 2} .
$$

Then $g$ is rank-one convex.

Proof. We have $H\left(a_{11}, a_{22}\right)=a_{11} a_{22}$. The bilinear form $H$ is a smooth separately convex function and $\sup \left|\partial^{2} H / \partial x \partial y\right|=1$. The assertion now follows easily from Lemma 5.1 of Šverák [8].

Now the question is whether the function (4.1) is quasiconvex. The answer is negative, as shown by the following lemma.

LEMmA 4.2. The function $g$ defined by (4.1) is not quasiconvex at the point

$$
B=\left[\begin{array}{cc}
1 & -1 \\
1 & 0
\end{array}\right]
$$

P r o of. We see that $g(B)=2$. Consider the function

$$
\varphi\left(x_{1}, x_{2}\right)=\left(\sin \pi x_{1}, \sin \frac{3 \pi}{2} x_{2}\right) .
$$

Then we have

$$
\nabla \varphi(x)=\left[\begin{array}{cc}
\pi \cos \pi x_{1} & 0 \\
0 & \frac{3 \pi}{2} \cos \frac{3 \pi}{2} x_{2}
\end{array}\right]
$$

Hence

$$
B+\nabla \varphi(x)=\left[\begin{array}{cc}
1+\pi \cos \pi x_{1} & -1 \\
1 & \frac{3 \pi}{2} \cos \frac{3 \pi}{2} x_{2}
\end{array}\right] \text {. }
$$

Thus

$$
g(B+\nabla \varphi(x))=2+\frac{3 \pi}{2} \cos \frac{3 \pi}{2} x_{2}+\pi \cos \left(\pi x_{1}\right) \frac{3 \pi}{2} \cos \frac{3 \pi}{2} x_{2} .
$$

Consequently, we get

$$
\begin{aligned}
\int_{[0,1]^{2}} g(B+\nabla \varphi(x)) d x= & 2+\int_{0}^{1} \frac{3 \pi}{2} \cos \frac{3 \pi}{2} x_{2} d x_{2} \\
& +\int_{[0,1]^{2}} \pi \cos \left(\pi x_{1}\right) \frac{3 \pi}{2} \cos \frac{3 \pi}{2} x_{2} d x_{1} d x_{2} \\
= & 2-1+0=1
\end{aligned}
$$


and

$$
\int_{[0,1]^{2}} g(B+\nabla \varphi(x)) d x=1<2=g(B) .
$$

By Lemma 2.4 we conclude that $g$ is not quasiconvex at $B$.

\section{References}

[1] E. Acerbi and N. Fusco, Semicontinuity problems in the calculus of variations, Arch. Rational Mech. Anal. 86 (1984), 125-145.

[2] J. M. Ball and F. Murat, $W^{1, p}$-quasiconvexity and variational problems for multiple integrals, J. Funct. Anal. 58 (1984), 225-253.

[3] P. G. Ciarlet, Mathematical Elasticity, Vol. 1: Three-Dimensional Elasticity, NorthHolland, Amsterdam, 1988.

[4] B. Dacorogna, Direct Methods in the Calculus of Variations, Springer, Berlin, 1989.

[5] B. Dacorogna and J. P. Haeberly, Remarks on a numerical study of convexity, quasiconvexity, and rank-one convexity, in: Progr. Nonlinear Differential Equations Appl. 25, Birkhäuser, Basel, 1996, 143-154.

[6] C. B. Morrey, Multiple Integrals in the Calculus of Variations, Springer, Berlin, 1966.

[7] P. Pedregal, Parametrized Measures and Variational Principles, Birkhäuser, Basel, 1997.

[8] V. Šverák, Rank-one convexity does not imply quasiconvexity, Proc. Roy. Soc. Edinburgh Sect. A 120 (1992), 185-189.

Université des Sciences et

Technologies de Lille

Avenue Paul Langevin

59655 Villeneuve d'Ascq, France
Institute of Fundamental Technological Research

Polish Academy of Sciences Świętokrzyska 21

00-049 Warszawa, Poland

E-mail: jtelega@ippt.gov.pl 